\title{
Study on the Rise and Fall of the Duyi Fu and Geography Record of the Pre-Tang Dynasty and The Mutual Influence
}

\author{
Tongen Li \\ College of Chinese Language and Literature of Northwest Normal University, Lanzhou, Gansu, 730070
}

Keywords: The Duyi Fu, The Geography Record, The Earthly Consciousness, The Literariness, The Mutual Influence

\begin{abstract}
There are two important regional works in the Tang Dynasty. As an important subject of Fu literature, it has a close relationship with the flourishing period of the Wei, Jin and Southern and Northern Dynasties. Both have a common origin, but also has a different development track; both self-contained, but also mutual influence. The artistic nature of the city and the nature of the material are permeated with each other, and the development and new changes are promoted.
\end{abstract}

\section{Introduction}

Duyi Fu, including Jingdu Fu and Yi Ju Fu, began in the Western Han Dynasty, to Yang Xiong "Shu Du Fu" as the earliest. Xiao Tong "anthology" received more than 90 articles, divided into 15 categories, the first category of "Kyoto", income 8; Li Fang and others compiled "Wen Yuan Yinghua" sub-43, "Kyoto" Yi "in the head; Chen Yuan-long series of" calendar Fu "column" city "head, a total of 10 volumes of 70 articles. Duyi Fu mainly revolves around the geography, politics, culture and social life of the metropolis,used for singing praises of the emperor and boast of their homelands, with obvious geographical characteristics.

Geography Record also known as the geography, geography or geography book, "Sui Shu - books catalogue" called it "geography". When the Western Han Dynasty into the emperor, "Liu Xiang sketched out the ground points, the prime minister Zhang Yu conmanded Yingchuan Zhu Jiang to record the customs", Liu Xiang, Zhu Gan has opened Geography Record written precedent. Since the Guangwu Emperor Liu Xiuzhao "Nanyang customs", three auxiliary, the Central Plains, Qilu and other places to write Geography Record become common practice. "Sui Shu - books catalogue" recorded geography book one hundred thirty-nine, one thousand four hundred and thirty volumes, the number of times after the miscellaneous, the second in the history of the Department. Most of the first Geography Record has been lost, today about 200 species of the works can be checked. Qing Dynasty Wang Mo "Geography Book notes from Han to Tang Dynasties" and today's people Liu Weiyi "Local Chronicles compilation from Han to Tang dynasties" embody a lot of lost texts.

\section{The Rise of Duyi Fu and Geography Record}

Duyi Fu has a common origin with the rise of Geography Record. Although the unified empire appeared late, but the unity concept of "All this territory belongs to the king" in the Western Zhou Dynasty has been generally popular. At the same time, people's state consciousness is more and more clear, "Nine states" "Twelve states"appear in"The Book of History",and this unified concept of state consciousness is Duyi Fu and Geography Record generated basis. "Zhou Guan" "Yu Gong" "Shan Hai Jing" and other early geographical works for the Duyi Fu and Geography Record prepared to prepare the prerequisites. Mr. Xu Jie believes that the early geography of the "Kyushu" pavement directly affect the formation of Fu body. Geography Record is based on early geography.

Although Duyi Fu and Geography Record have a common origin, but the direct reasons of the 
rise are different.

From the development process of $\mathrm{Fu}$, Duyi $\mathrm{Fu}$ is the result of the expansion of the subject matter in the process of development. The middle of the Western Han Dynasty, to Sima Xiangru's "Zi Xu Fu" "Shang Lin Fu" as the representative of the Hun hunting marks the maturity of the Han $\mathrm{Fu}$ and heyday. "Shu Du Fu" is Yang Xiong simulation "Zi Xu Fu" "Shang Lin Fu" made, to the United States and its state, boast of the native land. Although Yang Xiong has opened this end thread, but the main body of the Han Dynasty Duyi Fu Du Du's "Lun Du Fu" as the first pioneer of the Fu. This kind of Duyi Fu is to meet the needs of political unity arising from, and thus reached prosperity.

Around the Eastern Han Dynasty dispute, resulting in a number of political meaning of the strong Duyi Fu, especially to lay the status of Duyi Fu "Ode on Two Capitals" "The Prose Er Jing" is representative. With the Western Han Dynasty, "Zi Xu Fu" "Shang Lin Fu" and other Fu line, to praise the emperor's martial arts martial arts, supplemented by satirical encyclical. Zhang Heng's "South are Fu" is to write their own hometown, but the political color is also very heavy, because Nanyang is the home of the Eastern Han Dynasty Guangwu where he get on in life. Du Du "Lun Du Fu", Fu Yi "anti-Du Fu" "Luo Du Fu", Cui Yin "anti-Du Fu", Ban Gu "Ode on Two Capitals", Zhang Heng "The Prose Er Jing", are around the capital The problem started, and its purpose is to maintain a unified pattern. The Eastern Han Dynasty Fu family successively, and gradually established Duyi Fu creation of the paradigm, Duyi Fu Han Fu became an important theme.

Geography Record originated in the Han Dynasty, in the early geographical class works and metropolitan geography of the basis of the book do not one. Prosperous in the Han Dynasty weft, such as "Dun Jia Kai Shan Tu" "River Map" "Luo book" "River map of the territory" and so on, are often regarded as no talk. In fact, a class of geography of a kind of weft saved a lot of ancient geographical information, recorded a large number of mountains and rivers Yue ditch, such as Yunyang Mountain, Qiuyishan, Lishan, buried hill, Huoshan, Longxi Shenma Mountain, Baimashan, Qishan, stone Gushan, Lishan and so on. Compared to the weft book, Geography Record in the legendary color is not much better. It can be said that weft book is an important part of the history of Geography Record development.

Geography Record of the Han Dynasty more than the lost, only a few are only residual articles, and mostly written in the Eastern Han Dynasty. I know the Xin "Sanqin Record", Wang Bao "Yunyang Record", anonymous "Nanyang customs Record", Lu Zhi "Jizhou customs Record", Quan Cheng "Chenliu customs Record", Yang Zhong "Ailao Biography", Yang Fu"Jiao state Rarities Record "" Linhai water and soil Record "and so on. Geography Record in the state development at the same time, the national geography is also brewing. On the basis of the "Geography", the classics were organized into "Geography Section of History in Han Dynasty", and the national geography began to take shape, and its appearance also met the political needs of the Han Dynasty.

In general, Duyi Fu and Geography Record were two relatively independent systems in the Han Dynasty. Their development and maturity were not yet close.

\section{Duyi Fu and Geography Record Development}

The Eastern Han Dynasty, the political turmoil, tyrannical separatist, unified situation is no longer, people's territorial awareness increased. The original gathered in Kyoto, literati talent flow to the local, according to Haoqiang to self-protection, Fu description of the object moved from Kyoto to the local city, such as Xu dry "Qi Du Fu", Liu Zhen's "Lu Du Fu", Liu Shao's "Zhao Du Fu" and so on. These assignments are basically from the hands of their own people, boast side of the city, each to the name of Dayi own, competing feelings overflowing in the line between the lines.

Along the Han Dynasty Duyi Fu's creative mode, Zuo Si will be exaggerated play to the extreme. From the formal point of view, "Fu on Three Capitals" is the "Ode on Two Capitals" "The Prose Er Jing" followed, Han Fu Fu's style is not slightly changed, but the length has lengthened, the content more informative. Class, Zhang established the Duyi Fu paradigm in Zuo 
Si's pen to reach the peak, has been difficult to be beyond, no wonder later Xie criticized Yu "Yang $\mathrm{Du} \mathrm{Fu}$ " is "under the house."

Since the Wei and Jin Dynasties, Duyi Fu in the twists and turns in the development, and the occurrence of new changes - by the pride to turn to focus on lyrical, the system and the length has also changed. Ruan Ji's "Dong Ping Fu", "Yuan Fu Fu", a change "tellers to lead the first" of the system and praise the Du Yi Fu praised the traditional, space than the Han Dynasty Duyi Fu much smaller. He will Duyi Fu description of the object from Tongdu Dayi to a small town, and the two Han Duyi Fu Chen boasted the name of the bustling, mountains and rivers of the opposite of the property, he wrote is poor, dirty, remote, barren land, the people stubborn Clamor and so on ugly side. His purpose is not to lay out Hyun technology, but to express the heart of loneliness, pain and resentment.

During the Wei and Jin Dynasties, the local consciousness of the scholar was soaring, but the long-term war caused the city to be defeated, so that the Duyi Fu, with the aim of extolling, could not be sustained. "Local tyranny landlord politics, the expansion of economic power, the formation of the valve system" for the development of Geography Record created the conditions. The rise of the history of private history, pioneered by the literati, has played an important role in promoting the development and development of Geography Record. The southward of the economic center of gravity provides a material basis for the development of Geography Record. The long-term war that began in the late Eastern Han Dynasty directly undermined the economic development system of the North (mainly the Yellow River basin) and forced the northern population to move southward. In a relatively stable political situation, the Noble Manor economy is in the southern region of the rapid rise. The development of Jiangnan economy and the consolidation and development of political and cultural forces in Jiangnan area have aroused the attention of scholars to the unique geographical environment, property and customs of Jiangnan area. Therefore, the special record of the "Rarities Record" is very popular.

After the Migration Southwards of Yongjia Years, the Eastern Jin Dynasty is content to a part of the country, the mentality of the mentor changes. The development of the righteousness of mind in the river left, "deep into the life of all aspects of the scholar, affect their ideal of life, life, lifestyle, affect their aesthetic taste." The Eastern Jin Dynasty, the minister of the main weak, repeated riots occur, the scholar to escape the reality into the landscape of nature, the pursuit of quiet spiritual world. The beautiful scenery of the south has become a great place for the literati to escape from the world, and they are in love with the landscape, or by the love of the rain, or the hills of the mountains, and with the unprecedented enthusiasm to put into the writing of Geography Record. This style of the Nobles promoted the prosperity of Geography Record and influenced the entire Northern and Southern Dynasties.

Wei Jin Southern and Northern Dynasties nearly four hundred years time, the war is frequent, the regime change, although there is a brief reunification, but basically in the endless war and long-term division, which to some extent strengthened people's geographical consciousness With the idea. As a result, the Geography Record, which is dedicated to the corner of the city, has flourished for a long time. Unrest in the political situation, unyielding after the cultural self-esteem, it results in the prevalence of competing praies, the legendary corner, each other to win. Southern Liang Renfang compiled "Geography Record" two hundred and fifty-two volumes, which shows the rise of Geography Record in the Six Dynasties period.

Unlike the continued prosperity of Geography Record, Duyi Fu in the Northern and Southern Dynasties was declining, not only a few, but also less famous. The endless war and regime change, resulting in the public creatures, the city was a great degree of destruction, everywhere destroyed the scene, from Bao Zhao's "Rhapsody on Wucheng" can be seen. Warlord devastated Guangling City desolate, cold pressing, and the former mountains and plains, the city bustling in sharp contrast. The author tried to scream, loudly complain, but helpless, in this desperate mood, implies a strong desire for national unity.

Long wars to the people bear their sufferings, they hope that the early end of the split, return to unity. Geographers with this desire and aspirations, the integration of regional Geography Record 
compiled into a national geography, to Kan "Thirteen states Record" and Li Daoyuan "Commentary on the Waterways Classic" note the greatest impact. Under the pattern of regime and division of the north and the south, they break the regional limits and regard the confrontational North-South regime as an indivisible whole, which reflects the desire of the people of all ethnic groups across the country to have a profound political and historical meaning.

\section{The Mutual Influence of Duyi Fu and Geography Record}

Throughout the Han, Wei and Six Dynasties, Duyi Fu's literariness and Geography Record's pervasiveness blend and penetrate each other in the development of Duyi Fu and Geography Record, and promote the development and new development of each other.

First of all, Duyi Fu on a region of Geography Record development has a lasting impact, so that the state Geography Record to form a series of continuous works. A case study of Shu, for example, Yang Xiong "Shu Du Fu" first out, Qiao Zhou "three Pa Record" "Bashu Rarities Record", Chang Qu "Ba Han Record" "Shu Record" "The Records of State-Huayang " Yuan Xiuming "Bashu Record", any pre-, Li Ying and other families "Yizhou Record"appear successively in Shu.Others such as Luoyang Geography Record, Qi Geography Record,Wu Geography Record, Yangzhou Geography Record, etc., are so.

Second, Duyi Fu's content for the Geography Record books cited. Such as "Three Auxiliary Yellow Map" cited "Xidu Fu" 3, "Xijing Fu" 7. Li Daoyuan "Commentary on the Waterways Classic" cited Yang Xiong "Shu Du Fu" 5, Zhang Heng "Xijing Fu" 3, "Dongjing Fu" 4, "South Du Fu" 6, "Qi Du Fu" 4, "Lu Du Fu "1, Liu Shao" Zhao Du Fu "1, Zuo Si" Fu on Three Capitals"6, Yuchan" Yang Du Fu "1.

Thirdly, an important aspect of the influence of Duyi Fu to Geography Record is that the literary enhancement of Geography Record. Although the awakening of the Wei and Jin dynasties caused the consciousness of the literature, literary aesthetics by the unprecedented attention, this trend reflects the development of a variety of styles. However, the enhancement of the literary color of Geography Record is inseparable from the direct influence of Duyi Fu. This is mainly reflected in the landscape Geography Record on the scenery, such as Luohan "Xiangzhong Record":

Sunshine illuminates Xiang river into five or six Zhang, the stones at the bottom of the river look like go chess pieces, bright colors, white sands such as frost and snow, red cliff seems the glow.

Pensile Springs(Waterfall) on Mountain Heng, dripping from rocks, the sound Ling Ling, sounds like music of a stringed instrument, just as a crane hover over it , dancing with the wind.

The same is true of other Records ,such as Yuan Shan Song "Yidu mountains and rivers Record", Sheng Hongzhi "Jingzhou Record" and so on, in view of the objective things itself gives vivid description of the different forms, static and dynamic, bright and beautiful, literariness enhancement, obviously affected by Duyi Fu.

First, the real and detailed nature of the Geography Record content affects Du Yi Fu's lethality. Geography Record has miscellaneous memory, the characteristics of the museum, the history, geography, territory, milage, mountains, monuments, architecture, people, customs, nations, property and other content, mainly levying. Zuo Si "Fu on Three Capitals" and predecessors of the quite different, that is, he absorbed, drawing on a lot of Geography Record content. By the fictional tend to be true, this is Duyi Fu new changes and a feature. Although exaggerated elements for the purpose of literary description still exist, the written name is more than one real. Zuo Si bluntly is "Fu on Three Capitals", "mountains and towns is on the basis of the map, birds and animals and vegetation is in the light of the chronicles. Folk songs and dances, each attached to its customs, burly elders, without exception is old."

Second, Geography Record in turn became the object of Duyi Fu. By the influence of Duyi Fu, Geography Record in the Wei and Jin Dynasties was developing rapidly. There were a lot of geography records in the state of the Wei and Jin Dynasties. There was a lot of Geography Record. The emergence of Geography Record and the states culture exchange provide large amounts of reference material for prosody composers. 


\section{Conclusion}

The relatively unified political situation and the "united" ideology of the Han dynasty promoted the rapid maturity of Duyi Fu, and Duyi Fu achieved great achievements, while Geography Record, which emerged from the Han Dynasty, was in a slow development. Duyi Fu and Geography Record are relatively independent, and the relationship is not close to each other. During the Wei and Jin Dynasties and Northern and Northern Dynasties, Geography Record was developed rapidly, reaching its heyday and occupying the mainstream position of local writings. Duyi Fu gradually faded. In the Wei and Jin Dynasties, Duyi Fu interacted with Geography Record. On the one hand, Duyi Fu gave birth to a large number of geography records. On the other hand, the rise of Geography Record also provided a great deal of material for Duyi Fu's creation and contributed to the development of Duyi Fu With new change. Whether it is a brief decline in Duyi Fu, or the continued prosperity of Geography Record, are implicit in their new budding.

\section{References}

[1] (Tang) Wei Zheng: "Sui Shu ", Zhonghua Book Company in 1973, the first 988 pages.

[2] Zhu Gan's "Geography", also known as "Customs", see Wang Yong "History of Chinese geography", the Commercial Press in 1984.

[3] Wang Lin: "Six Dynasties Geography Record : the combination of geography and literature", "Literature and History" 2012 the first period.

[4] "The Book of Songs • Xiaoya • Beishan".

[5] "The Book of History • Shun Code": "There were twelve states from the start."

[6] Xu Jie: "Geography of the Qing Dynasty and Jiangyu Fu", "Chinese books and culture" 1995 the first period.

[7] (Song of the Southern dynasties) Liu Yiqing, (Liang of the Southern dynasties) Liu Xiaobiao Annotate, Yu Jiaxi Jian Comment: "New Anecdotes of Social Talk Jian Shu", Zhonghua Book Company 2015, the first 284 pages.

[8] Cang Xiuliang: "Theory of Chorography", East China Normal University Press, 2014, p. 84.

[9] Luo Zongqiang: "Metaphysics and Wei Jin scholar mentality", Tianjin Education Press 2005, p. 234.

[10] (Wei of the Northern dynasties) Li Daoyuan, Chen Qiaoyi Correct: "Water by the school card", Zhonghua Book Company 2007, the 896-897 pages.

[11] (Tang) Xu Jian ,etc: "Chu Xue Ji", Zhonghua Book Company 1962, p. 98.

[12] (Liang of the Southern dynasties) Xiao Tong, (Tang) Li Shan Annotate: "Anthology", Zhonghua Book Company 1986, p. 174. 